\title{
Dynamics of a large extra dimension inspired hybrid inflation model
}

\author{
Anne M. Green \\ Astronomy Unit, School of Mathematical Sciences, Queen Mary, University of London, Mile End Road, London E1 4NS, United Kingdom \\ and Physics Department, Stockholm University, S-106 91, Stockholm, Sweden* \\ Anupam Mazumdar \\ The Abdus Salam International Centre for Theoretical Physics, I-34100, Trieste, Italy
}

(Received 24 January 2002; published 15 May 2002)

\begin{abstract}
In low scale quantum gravity scenarios the fundamental scale of nature can be as low as $1 \mathrm{TeV}$, in order to address the naturalness of the electroweak scale. A number of difficulties arise in constructing specific models: stabilization of the radius of the extra dimensions, avoidance of overproduction of Kaluza-Klein modes, achieving successful baryogenesis and production of a close to scale-invariant spectrum of density perturbations with the correct amplitude. We examine in detail the dynamics, including radion stabilization, of a hybrid inflation model that has been proposed in order to address these difficulties, where the inflaton is a gauge singlet residing in the bulk. We find that for a low fundamental scale the phase transition, which in standard four dimensional hybrid models usually ends inflation, is slow and there is a second phase of inflation lasting for a large number of $e$-foldings. The density perturbations on cosmologically interesting scales exit the Hubble radius during this second phase of inflation, and we find that their amplitude is far smaller than is required. We find that the duration of the second phase of inflation can be short, so that cosmologically interesting scales exit the Hubble radius prior to the phase transition, and the density perturbations have the correct amplitude, only if the fundamental scale takes an intermediate value. Finally we comment briefly on the implications of an intermediate fundamental scale for the production of primordial black holes and baryogenesis.
\end{abstract}

DOI: 10.1103/PhysRevD.65.105022

PACS number(s): $11.10 . \mathrm{Kk}, 12.90 .+\mathrm{b}, 98.80 . \mathrm{Cq}$

\section{INTRODUCTION}

In nature there are two apparent scales: the electroweak scale and the scale of gravity, separated by 17 orders of magnitude. Understanding the gap between these scales has been a prime motivation behind studying theories beyond the electroweak standard model (SM). Supersymmetry provides an elegant scheme for keeping the electroweak scale stable under any large radiative corrections; however, the lack of direct evidence for supersymmetry in collider physics and in nature has led to the consideration of scenarios with large extra dimensions. In these scenarios the fundamental scale is taken to be the higher dimensional Planck mass $M_{*}$, which is assumed to be close to the electroweak scale $[1,2]$. While in this scheme supersymmetry is redundant in four dimensions, the presence of low energy supersymmetry could still be a viable option, however, with the fundamental scale at an intermediate scale, somewhere between the Planck and electroweak scales. Such a scenario is well motivated by string theory [3], which predicts that gauge and gravity unification occurs below the grand unification scale $\sim 10^{16.5} \mathrm{GeV}$.

The four dimensional Planck mass in these theories is obtained via dimensional reduction, assuming that the extra dimensions are compactified on a torus, the simplest possible manifold. The volume of the extra dimensions $V_{d}$, the effective four dimensional Planck mass and the fundamental scale are then simply related:

$$
M_{\mathrm{p}}^{2}=M_{*}^{2+d} V_{d},
$$

*Present address. where $d$ is the number of extra compact dimensions. For given $M_{*}$ this fixes the present day size of each of the extra dimensions, $b_{0}$. For two extra dimensions and $M_{*}$ $\sim 1 \mathrm{TeV}, b_{0} \sim 0.2 \mathrm{~mm}$. Currently collider physics and supernovas 1987A impose a bound on the fundamental scale, $M_{*} \geqslant 30 \mathrm{TeV}[1,4,5]$, and a recent astrophysical bound based on a heating of a neutron star suggets $M_{*}$ $\geqslant 500 \mathrm{TeV}$ [9]. If the fundamental scale is as low as $\sim 100 \mathrm{TeV}$, it is important that the SM particles are trapped in a four dimensional hypersurface (a 3-brane) and are not allowed to propagate in the bulk [1]. It is generically assumed that besides gravity, the SM singlets, which may include the inflaton, can propagate in the bulk [6].

The cosmological setup in models with large extra dimensions is quite different from the conventional one. First, if the electroweak scale is the fundamental scale in higher dimensions, then there can be no massive fields beyond the electroweak scale in four dimensions. Second, the size of the extra dimensions can be quite large, compared to the electroweak scale, which implies the existence of new degrees of freedom, usually known as the radion, with a mass scale as small as $\mathcal{O}(0.01 \mathrm{eV})$ if there are two large extra dimensions. The large extra dimensions must grow from their natural scale of compactification, $\sim 1(\mathrm{TeV})^{-1}$, and then stabilize at around a millimeter. This stabilization must occur before the electroweak phase transition and nucleosynthesis, via some kind of a trapping mechanism as discussed in Ref. [7]. The Kaluza-Klein (KK) states of the graviton, and any other fields residing in the bulk, can be excited at high temperatures and hence lead to constraints on these models. Above the normalcy temperature, the universe could be filled by the KK modes. For big bang nucleosynthesis to occur success- 
fully the normalcy temperature must be greater than $\sim 1 \mathrm{MeV}$. Furthermore, the final reheat temperature, which is constrained by cosmological considerations to be as small as $100 \mathrm{MeV}[1,8,10]$, should be smaller than the normalcy temperature. These considerations severely restrict baryogenesis in these models; for a detailed discussion, see Refs. $[11,10]$.

Constructing a successful inflation model, which produces a close to scale invariant spectrum of density perturbations with the correct amplitude and a very low reheat temperature is a challenging issue, with single field models and models where the inflaton is a brane field proving particularly problematic $[12,13]$. There have been several proposals $[12,13]$, arguably the most natural of which invokes SM singlet scalars coupled together to form a potential which mimics that of the standard four dimensional hybrid inflationary model, but with the fields promoted to the higher dimensions [14,7]. It has also been shown that baryogenesis can occur successfully in this model $[11,10]$. In this paper we study the dynamics of this extra dimension inspired hybrid inflationary model in detail.

In hybrid inflation models, the false vacuum field is initially trapped in a stable minimum at zero while the inflaton field slow-rolls down its potential. At some critical value of the inflaton field the stable minimum becomes an unstable maximum and quantum diffusion produces a second order phase transition from the false vacuum to the true vacuum [15]. In standard four dimensional cosmology, for most parameter values, the bare mass of the false vacuum field is much greater than the Hubble parameter and the phase transition occurs rapidly and inflation ends. If these quantities have roughly the same magnitude, however, then the rolldown of the false vacuum field is no longer fast and a second period of inflation occurs $[16,17]$. In standard four dimensional cosmology, for the phase transition to occur slowly the effective coupling of the false vacuum field has to be tiny, $\sim 10^{-30}$ [17]. We find, however, that for the parameter values which are relevant for the extra dimensional model (fundamental coupling constants of order unity and fundamental scale $\sim 100 \mathrm{TeV}[14,7]$ ), the bare mass of the false vacuum field is of order the Hubble parameter so that the phase transition is slow and a second period of inflation occurs.

The duration of this second phase of inflation is typically very long, so that the density perturbations on cosmological scales are generated close its end. We calculate the amplitude of these perturbations and find that they are only compatible with the Cosmic Background Explorer (COBE) normalization if the fundamental scale is significantly larger than 100 TeV. Finally we discuss the implications, specifically achieving successfully baryogenesis and avoiding the overproduction of primordial black holes (PBHs), of an intermediate fundamental scale. In order to keep our discussion as general as possible, we do not fix either the fundamental scale or the number of extra dimensions from the outset; however, we will focus throughout on the parameter values of the specific model proposed in Refs. [14,7].

\section{MODEL AND ITS DYNAMICS}

A single field inflationary model, either in four dimensions or with the inflaton promoted into the bulk, cannot provide adequate density perturbations [14]. This has led to the suggestion of a hybrid inflationary model in higher dimensions with potential [14]

$$
V(\hat{N}, \hat{\phi})=\lambda^{2} M_{*}^{d}\left(N_{0}^{2}-\frac{1}{M_{*}^{d}} \hat{N}^{2}\right)^{2}+\frac{m_{\phi}^{2}}{2} \hat{\phi}^{2}+\frac{g^{2}}{M_{*}^{d}} \hat{N}^{2} \hat{\phi}^{2},
$$

where $\hat{\phi}$ is the inflaton field and $\hat{N}$ is the subsidiary false vacuum field which is responsible for the phase transition. The coupling constants, $g$ and $\lambda$, need not be identical, but we assume them to be of $\mathcal{O}(1)$. The four dimensional Higgs vacuum expectation value is determined by $\lambda N_{0}$, and should be of order $\sim \mathcal{O}(100) \mathrm{GeV}$. The higher dimensional field has a mass of dimension $1+d / 2$, which leads to nonrenormalizable interaction terms. The suppression, however, is given by the fundamental scale, instead of the four dimensional Planck mass. Upon dimensional reduction the effective four dimensional fields, $\phi$ and $N$, are related to their higher dimensional cousins by a simple scaling:

$$
\phi=\sqrt{V_{d}} \hat{\phi}, \quad N=\sqrt{V_{d}} \hat{N} .
$$

From the point of view of four dimensions the extra dimensions are assumed to be compactified on a $d$ dimensional Ricci flat manifold with radii $b(t)$, with a minimum at $b_{0}$. The higher dimensional metric then reads

$$
d s^{2}=d t^{2}-a^{2}(t) d \vec{x}^{2}-b^{2}(t) d \vec{y}^{2}
$$

where $\vec{x}$ denotes the three spatial dimensions, and $\vec{y}$ collectively denotes the extra dimensions. The scale factor of the four dimensional space-time is denoted by $a(t)$. After dimensional reduction the effective four dimensional action reads

$$
\begin{aligned}
S= & \int d^{4} x \sqrt{-g}\left[-\frac{M_{\mathrm{p}}^{2}}{16 \pi} R+\frac{1}{2} \partial_{\mu} \sigma \partial^{\mu} \sigma-U(\sigma)+\frac{1}{2} \partial_{\mu} \phi \partial^{\mu} \phi\right. \\
& \left.+\frac{1}{2} \partial_{\mu} N \partial^{\mu} N-\exp \left(-d \sigma / \sigma_{0}\right) V(\phi, N)\right]
\end{aligned}
$$

where the potential $V(\phi, N)$ can be derived from Eqs. (2) and (3),

$$
\begin{aligned}
V(\phi, N) \equiv & \left(\frac{M_{\mathrm{p}}}{M_{*}}\right)^{2} \lambda^{2} N_{0}^{4}+\frac{\lambda^{2}}{4}\left(\frac{M_{*}}{M_{\mathrm{p}}}\right)^{2} N^{4}-\lambda^{2} N_{0}^{2} N^{2} \\
& +g^{2}\left(\frac{M_{*}}{M_{\mathrm{p}}}\right)^{2} \phi^{2} N^{2}+\frac{1}{2} m_{\phi}^{2} \phi^{2},
\end{aligned}
$$

and has a global minima at

$$
\phi=0, \quad N^{2}=2\left(\frac{M_{\mathrm{p}}}{M_{*}}\right)^{2} N_{0}^{2} .
$$

In Refs. [14,7] the parameter values $N_{0}=M_{*} \sim 10^{5} \mathrm{GeV}$, $m_{\phi} \sim 10 \mathrm{GeV}$ and $\lambda \sim g \sim 1$ were taken. 
The radion field $\sigma(t)$ can be written in terms of the radii of the extra dimension:

$$
\sigma(t)=\sigma_{0} \ln \left[\frac{b(t)}{b_{0}}\right], \quad \sigma_{0}=\left[\frac{d(d+2) M_{\mathrm{p}}^{2}}{16 \pi}\right]^{1 / 2} .
$$

Note that $\sigma_{0}$ is proportional to the four dimensional Planck mass. For illustrative purposes, if we take the fundamental scale $\sim \mathcal{O}(\mathrm{TeV})$, the natural size of our three spatial dimensions - and also the size of the extra spatial dimensions-is determined by the fundamental scale $a(t)$ $\sim b(t) \sim 1(\mathrm{TeV})^{-1}$. From Eq. (1), assuming that there are only two extra dimensions, the present size of the extra dimensions must be of order $b_{0} \sim \mathcal{O}(1 \mathrm{~mm})$. The size of $b(t)$ must therefore expand from $1(\mathrm{TeV})^{-1}$ and be stabilized at 1 $\mathrm{mm}$; i.e., there must be some mechanism which traps the radion field in the minimum of the radion potential, $U(\sigma)$. There is no concrete origin for this potential; however, the simplest possibility which gives the correct mass for the radion is $U(\sigma) \sim m_{r}^{2} \sigma^{2}$, where $m_{\mathrm{r}} \sim 10^{-2} \mathrm{eV}$ for two extra spatial dimensions. A mechanism which can trap the radion field in its potential was provided in Ref. [7] and we will now discuss its dynamics in detail.

Initially the dynamics of the universe are dominated by the exponential potential of the radion. If $g N_{0} \gg m_{\phi}$, the false vacuum term dominates Eq. (6): $V(\phi, N)$ $\approx \lambda^{2}\left(M_{\mathrm{p}} / M_{*}\right)^{2} N_{0}^{4} \approx$ const. The exponential term, due to the radion field, multiplying the constant scalar potential in Eq. (5) leads to a period of power law asymmetric expansion for $a(t)$ and $b(t)[18,13,7]$ :

$$
a(t) \sim t^{(d+2) / d}, \quad b(t) \sim t^{2 / d} .
$$

From the four dimensional point of view the radion drives a period of inflation as it rolls down the exponential potential. Once it reaches the critical value $\left|\sigma_{0}\right|$ [see Eq. (5)] the effective mass of the radion field becomes of the order of the Hubble parameter:

$$
m_{\mathrm{r}, \mathrm{eff}}^{2} \approx m_{\mathrm{r}}^{2}+\frac{V(\phi, N)}{\sigma_{0}^{2}} \sim \mathcal{O}(1) H^{2},
$$

where we have neglected the contribution from $U(\sigma)$ as it is small compared to that from $V(\phi, N)\left[U(\sigma) \sim M_{\mathrm{p}}^{2} m_{\mathrm{r}}^{2}\right.$ $\ll V(\phi, N) \sim M_{\mathrm{p}}^{2} M_{*}^{2}$, as $\left.\sigma(t) \rightarrow \sigma_{0} \approx M_{\mathrm{p}}\right]$. At this point the radion field can no longer support inflation; however, inflation continues as the $\phi$ field slowly rolls down the potential, with Hubble parameter

$$
H \approx \sqrt{\frac{8 \pi}{3}} \frac{\lambda N_{0}^{2}}{M_{*}} .
$$

Subsequently the mass of the radion field is dominated by the Hubble constant, $H$, and the radion field approaches the global minimum configuration, $\sigma=0$, exponentially fast:

$$
\sigma(t) \approx \sigma_{0} e^{\left(-m_{\mathrm{r}, \mathrm{eff}}^{2}(H) t / 3 H\right)} \sim \sigma_{0} e^{(-H t / 3)},
$$

so that the radion is trapped in its own potential $U(\sigma)$, within a Hubble time, and the radion configuration remains dynamically frozen while inflation continues. As the radion couples to the trace of the energy momentum tensor it therefore also couples to the SM particles, which are essentially the decay products of $\phi$ and $N$, and the Hubble induced correction remains even after the end of inflation. Note that the radion interaction with the SM fields is extremely weak, due to the Planck mass suppressed couplings, and hence it can never reach thermal equilibrium. At a certain energy scale, when the bare mass of radion mass comes to dominate the Hubble induced correction, the radion begins to oscillate around the minimum of the potential with an amplitude $\propto m_{\mathrm{r}}$. If the bare mass of the radion is very small, $\lesssim 10^{-2} \mathrm{eV}$ in the case of two large extra dimensions and the fundamental scale or of the order of $1 \mathrm{TeV}$, then the radion density stored in the oscillations is not large enough to act as a dominant component of the total energy density of the universe. However, for a mass as small as $10^{-2} \mathrm{eV}$, the oscillations are rapid, $\nu \sim m_{\mathrm{r}}^{-1} \sim 10^{11} \mathrm{~Hz}$, which suggests that Newton's constant may get a time varying contribution from the radion oscillations [19], so that $\dot{G} / G \neq 0$. In theories where Newtons constant is time varying, such as the Brans-Dicke theory of gravity [20], the time variation in $G$ contributes to the total energy density and therefore affects the expansion rate of the universe. Even though the amplitude of the radion oscillations is small, the high frequency of the oscillations may provide a significant contribution to the Hubble expansion. This is an interesting topic which merits a separate study.

We will now concentrate on the subsequent dynamics of the $\phi$ and $N$ fields. The $N$ field is rapidly driven to the false vacuum, $N=0$, and the $\phi$ field rolls slowly down the potential until it reaches the critical value, $\phi_{\mathrm{c}}$,

$$
\phi_{\mathrm{c}}=\frac{\lambda}{g}\left(\frac{N_{0} M_{\mathrm{p}}}{M_{*}}\right) \text {, }
$$

where the effective mass squared of the $N$ field becomes negative and a second order phase transition begins. Note that if $\lambda \sim g$ and $N_{0} \sim M_{*}$, then $\phi_{\mathrm{c}} \sim M_{\mathrm{p}}$. The effective mass of the false vacuum field, $m_{N}$, is

$$
m_{N}=\sqrt{2} \lambda N_{0},
$$

so that

$$
\frac{m_{N}}{H} \approx \sqrt{\frac{3}{4 \pi}} \frac{M_{*}}{N_{0}} .
$$

For $M_{*} \sim N_{0}, H \sim m_{N}$, so that the phase transition occurs slowly and there is a second period of inflation as the fields initially roll slowly towards the global minimum of the potential.

The dynamics of hybrid inflation models where there is a second period of inflation have previously been studied by Randall, Soljacić, and Guth [16] and García-Bellido, Linde, and Wands [17] (GLW), with particular focus on the production, at the phase transition, of large density perturbations, 
which may lead to the over-production of primordial black holes. GLW parametrize the potential as

$$
V(\phi, \psi)=\left(M^{2}-\frac{\sqrt{\tilde{\lambda}}}{2} \psi^{2}\right)^{2}+\frac{1}{2} \tilde{m}_{\phi}^{2} \widetilde{\phi}^{2}+\frac{1}{2} \gamma \widetilde{\phi}^{2} \psi^{2}
$$

and find that $m_{\phi} \sim H \sim 1 \mathrm{TeV}$ if $M \sim 10^{11} \mathrm{GeV}, m$ $\sim 10^{3} \mathrm{GeV}$ and $\lambda \sim 10^{-30}$. Comparing Eqs. (6) and (16) one finds the following parameter equivalences: $\phi \equiv \widetilde{\phi}, m_{\phi}$ $\equiv \tilde{m}_{\phi}$,

$$
\begin{aligned}
& \frac{\lambda}{2}\left(\frac{M_{\mathrm{P}}}{M_{*}}\right) N_{0}^{2} \equiv M^{2}, \\
& \frac{g^{2}}{\lambda}\left(\frac{M_{*}}{M_{\mathrm{P}}}\right) \equiv \frac{1}{2} \frac{\gamma}{\sqrt{\tilde{\lambda}}}, \\
& \lambda\left(\frac{M_{*}}{M_{\mathrm{P}}}\right) N^{2} \equiv \sqrt{\tilde{\tilde{\lambda}} \psi^{2} .}
\end{aligned}
$$

So we see that, if $M_{*} \sim N_{0} \sim 10^{5} \mathrm{GeV}$, then we automatically obtain the extremely small effective coupling for the false vacuum field, required for a slow phase transition and second period of inflation, for values of the fundamental couplings $g$ and $\lambda$ of order unity. One important difference between our model and that of GLW is the critical value of the slow-rolling field. GLW have

$$
\widetilde{\phi}_{\mathrm{c}}^{2}=\frac{2 \sqrt{\lambda}}{\gamma} M^{2} \sim \frac{10^{-31}}{\gamma} M_{\mathrm{P}}^{2}
$$

whereas we have $\phi_{\mathrm{c}} \sim M_{\mathrm{P}}$. This suggests that in our case the dynamical behavior of the fields is quite different from that of previously studied hybrid inflation models, even those where a slow phase transition occurs $[16,17]$.

We will now study the dynamics of the fields for our parameters analytically. Once the radion has stabilized and the false vacuum field $N$ has evolved to $N=0$, provided that $g N_{0} \gg m_{\phi}$ the false vacuum term dominates the potential, so that the evolution of the $\phi$ field is given by

$$
\phi=\phi_{\mathrm{i}} \exp \left(-\frac{1}{\sqrt{6 \pi} \lambda} \frac{M_{*} m_{\phi}^{2}}{N_{0}^{2}} t\right),
$$

where $\phi_{\mathrm{i}}$ is the initial value at some arbitrary initial time $t$ $=0$. The number of $e$-foldings of inflation which occur between $\phi_{\mathrm{i}}$ and $\phi$ is given by

$$
N_{\mathrm{e}}=2 \pi \lambda^{2} \frac{N_{0}^{4}}{M_{*}^{2} m_{\phi}^{2}} \ln \frac{\phi_{\mathrm{i}}}{\phi} .
$$

We see that for $N_{0} \sim M_{*} \gg m_{\phi}$ the evolution of the $\phi$ field is extremely slow, and the duration of this first phase of inflation is large.

The evolution of $N$ as $\phi \rightarrow \phi_{\mathrm{c}}$ is more complicated, as $m_{N}^{2}<H^{2}$ once

$$
\phi^{2}<\phi_{\mathrm{c}}^{2}+\frac{4 \pi}{3}\left(\frac{\lambda N_{0}^{2} M_{\mathrm{P}}}{M_{*}}\right)^{2}
$$

so that the quantum fluctuations in the $N$ field become important. The Fokker-Planck equation [21] is usually employed to study the dynamics of the $N$ field in this regime $[16,17]$, using the assumptions that the field has a deltafunction distribution at some initial time (when $\phi \gg \phi_{c}$ ) and that the average quantum diffusion per Hubble volume per Hubble time is $\approx H / 2 \pi$. It was found in Ref. [17] that the typical value of the $N$ field when $\phi=\phi_{\mathrm{c}}, \bar{N}$, is given by

$$
\bar{N}^{2}=\left(\frac{H}{2 \pi^{2}}\right)^{2} \frac{1}{2 r}\left(\frac{e^{a}}{a}\right)^{a} \Gamma(a, a),
$$

where $a=\left(4 \lambda^{2} N_{0}^{2} / 3 m_{\phi}^{2}\right), \Gamma(a, a)$ is the incomplete gamma function and

$$
r=\frac{3}{2}-\sqrt{\frac{9}{4}-\frac{m_{\phi}^{2}}{H^{2}}} \approx \frac{m_{\phi}^{2}}{3 H^{2}} .
$$

Equation (22) must be evaluated numerically; for example, if $\lambda=g=1, \quad N_{0}=M_{*}=10^{5} \mathrm{GeV}$ and $m_{\phi}=10 \mathrm{GeV}$, as in Refs. [14,7], then $\bar{N}=22(H / 2 \pi)$.

In order to study the second phase of inflation which occurs after $\phi=\phi_{\mathrm{c}}$, it will prove useful to rewrite Eq. (6) using Eq. (13) as

$$
\begin{aligned}
V(\phi, N) \equiv & \left(\frac{M_{\mathrm{p}}}{M_{*}}\right)^{2} \lambda^{2} N_{0}^{4}+\frac{\lambda^{2}}{4}\left(\frac{M_{*}}{M_{\mathrm{p}}}\right)^{2} N^{4}+g^{2}\left(\frac{M_{*}}{M_{\mathrm{p}}}\right)^{2} N^{2}\left(\phi^{2}\right. \\
& \left.-\phi_{\mathrm{c}}^{2}\right)+\frac{1}{2} m_{\phi}^{2} \phi^{2} .
\end{aligned}
$$

Furthermore, if $N_{0} \gg m_{\phi}$, then for $N \ll\left(M_{\mathrm{P}} N_{0} / M_{*}\right)$ the false vacuum term in the potential dominates and the Hubble parameter remains constant.

The slope of the potential in the $\phi$ direction is given by

$$
\frac{\mathrm{d} V}{\mathrm{~d} \phi}=\left[2 g^{2}\left(\frac{M_{*}}{M_{\mathrm{P}}}\right)^{2} N^{2}+m_{\phi}^{2}\right] \phi .
$$

Provided that $\left(10 \lambda N_{0}^{2}\right) /\left(g m_{\phi} M_{\mathrm{P}}\right) \ll 1$, then the second term dominates initially and $\phi$ evolves away from $\phi_{\mathrm{c}}$ with equation of motion

$$
\phi=\phi_{c} \exp \left[-\frac{1}{\sqrt{24 \pi}} \frac{1}{\lambda}\left(\frac{m_{\phi}}{N_{0}}\right)^{2}\left(M_{*} \hat{t}\right)\right],
$$

where we have now taken $\hat{t}=0$ when $\phi=\phi_{\mathrm{c}}$.

In the $N$ direction the slope of the potential is given by

$$
\frac{\mathrm{d} V}{\mathrm{~d} N}=\left[\lambda^{2} N^{2}+2 g^{2}\left(\phi^{2}-\phi_{c}^{2}\right)\right] N\left(\frac{M_{*}}{M_{\mathrm{P}}}\right)^{2} .
$$

If $\bar{N}$ is sufficiently large [as is the case for the specific parameter values we are interested in, $\bar{N}=22(H / 2 \pi)]$, then 
there is a small period where the first term dominates and $N(\hat{t}) \sim \bar{N}$. As $\phi$ evolves away from $\phi_{\mathrm{c}}$, however, the second term soon comes to dominate, so that for small $\hat{t}$, using the first order expansion of Eq. (26),

$$
\phi-\phi_{c} \sim-\frac{1}{\sqrt{24 \pi}} \frac{1}{g}\left(\frac{m_{\phi}^{2}}{N_{0}}\right) M_{\mathrm{p}} t
$$

the $N$ field grows exponentially:

$$
N=\bar{N} \exp \left[\frac{1}{12 \pi}\left(\frac{m_{\phi}}{N_{0}}\right)^{2}\left(M_{*} t\right)^{2}\right],
$$

where we have neglected the initial period where $N \sim \bar{N}$ since its duration is negligible compared with that of the subsequent exponential growth. For the majority of the duration of the second phase of inflation the $N$ field grows exponentially and $\phi$ moves slowly away from $\phi_{\mathrm{c}}$. Once

$$
2 g^{2}\left(\frac{M_{*}}{M_{\mathrm{P}}}\right)^{2} N^{2} \sim m_{\phi}^{2}
$$

however, the first term in Eq. (25), which is growing exponentially, comes to dominate the evolution of the $\phi$ field and causes the $\phi$ field to evolve rapidly away from $\phi_{\mathrm{c}}$. At this point $N \sim\left(\phi_{\mathrm{c}}-\phi\right)$ so that $\mathrm{d} V / \mathrm{d} N \sim \mathrm{d} V / \mathrm{d} \phi$ and both $N$ and $\left(\phi_{\mathrm{c}}-\phi\right)$ grow rapidly, and inflation comes to an end shortly afterwards, with both fields subsequently oscillating about the global minimum of the potential. We can therefore use the time at which Eq. (30) is satisfied to estimate the duration of the second phase of inflation, $t_{2}$ :

$$
t_{2} \approx \frac{\sqrt{6 \pi} N_{0}}{m_{\phi} M_{*}}\left\{\ln \left[\frac{1}{2 g^{2}}\left(\frac{M_{P}}{M_{*}}\right)^{2}\left(\frac{m_{\phi}}{\bar{N}}\right)^{2}\right]\right\}^{1 / 2} .
$$

Since the Hubble parameter remains constant until very close to the end of the second phase of inflation, we can estimate the total number of $e$-foldings which occur during the second phase of inflation as

$$
N_{\mathrm{e} 2} \approx H t_{2}=4 \pi \lambda \frac{N_{0}^{3}}{M_{*}^{2} m_{\phi}} \times\left\{\ln \left[2 \sqrt{\pi}\left(\frac{N_{0}}{M_{*}}\right)^{2}\left(\frac{M_{\mathrm{p}}}{\bar{N}}\right)\right]\right\}^{1 / 2} .
$$

For the parameter values used in Refs. [14,7] $\left[N_{0} \sim M_{*}\right.$ $\sim 10^{5} \mathrm{GeV}, m_{\phi} \sim 10 \mathrm{GeV}$, and $\left.\lambda \sim g \sim \mathcal{O}(1)\right]$ we obtain $N_{\mathrm{e} 2} \sim 9 \times 10^{5}$, an extremely large number of $e$-foldings of inflation. This reiterates the point that for $N_{0} \sim M_{*}$ the phase transition is extremely slow.

This description is borne out by numerical evolution of the full equations of motion of the fields. We find that, for the set of parameter values above, the second phase of inflation lasts for $8.5 \times 10^{5} e$-foldings, and that $\phi \sim \phi_{\mathrm{c}}$ and $N \sim 0$ until the last $100 e$-foldings or so. In Fig. 1 we plot the evolution of the fields during the late stages of the second phase of inflation. Note that the evolution of the fields away from $\phi$ $=\phi_{\mathrm{c}}$ and $N=0$ at the end of the second phase of inflation is so rapid that if we plotted their evolution for the entire sec-

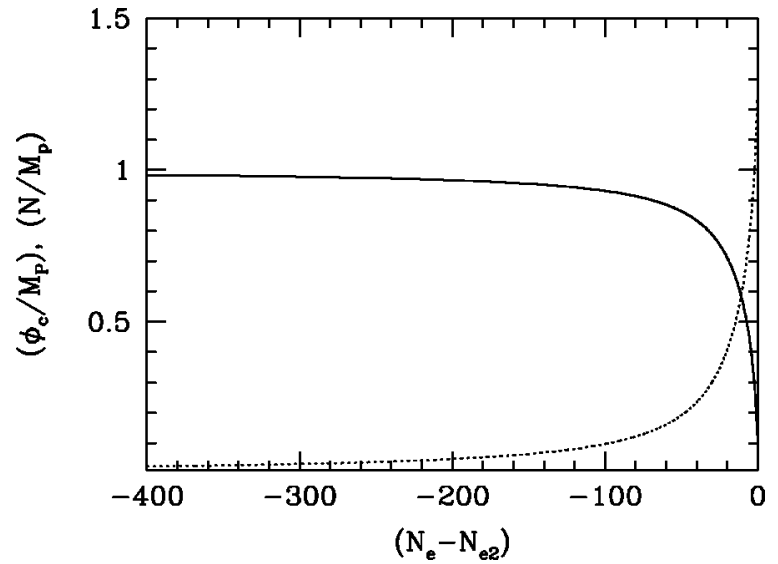

FIG. 1. The evolution of the $\phi$ (solid curve) and $N$ (dotted curve) fields as a function of minus the number of $e$-foldings from the end of inflation (so that time flows from left to right), towards the end of the second phase of inflation, for the parameter values $N_{0}=M_{*}=10^{5} \mathrm{GeV}, m_{\phi}=10 \mathrm{GeV}$, and $\lambda=g=\mathcal{O}(1)$, so that $N_{\mathrm{e} 2} \approx 8.5 \times 10^{5}$.

ond period of inflation linearly, only the straight lines $\phi$ $=\phi_{\mathrm{c}}$ and $N=0$ would be visible.

\section{DENSITY PERTURBATIONS}

In this model the scales relevant for the microwave background and large scale structure exit the Hubble radius 43 $e$-foldings before the end of inflation [14]; this is less than the usual $60 e$-foldings because of the small inflationary energy scale and the requirement that the reheat temperature should be $\sim \mathcal{O}(10-100) \mathrm{MeV}$, in order not to overproduce bulk matter such as excitation of KK modes (for details see Ref. [10]). The amplitude of the density perturbations on scales which leave the Hubble radius towards the end of the first period of inflation, when $\phi \sim \phi_{\mathrm{c}}$ and $N \sim 0$, can be calculated easily $[14,10]$ :

$$
\delta_{\mathrm{H}}=8.2 \lambda^{2} g \frac{N_{0}^{5}}{M_{*}^{2} m_{\phi}^{2} M_{\mathrm{p}}} .
$$

For $\lambda \sim g \sim 1, N_{0} \sim M_{*} \sim 10^{5} \mathrm{GeV}$, then to produce the COBE normalization, $\delta_{\mathrm{H}} \simeq 1.95 \times 10^{-5}$ [22], requires $m_{\phi}$ $\sim 10 \mathrm{GeV}[14,7]$; however we have already seen that for these parameter values $N_{\mathrm{e} 2} \sim 9 \times 10^{5} \gg 43$, so that the scales that left the Hubble radius during the first period of inflation have yet to re-enter the Hubble radius. In other words the scales which are cosmologically interesting leave the Hubble radius during the second phase of inflation. Combining Eqs. (32) and (33) we find that requiring the perturbations on the scales probed by COBE to leave the Hubble radius during the first phase of inflation and have the correct amplitude $\left(N_{\mathrm{e} 2}<43\right.$ and $\left.\delta_{\mathrm{H}}=1.9 \times 10^{-5}\right)$ requires

$$
\frac{M_{\mathrm{P}} N_{0}}{g M_{*}^{2}}\left[\ln \left\{2 \sqrt{\pi}\left(\frac{N_{0}}{M_{*}}\right)^{2}\left(\frac{M_{\mathrm{p}}}{\bar{N}}\right)\right\}\right] \lesssim 10^{6},
$$


so for $M_{*} \sim 10^{5} \mathrm{GeV}$ and $g \sim \mathcal{O}(1)$ we would need $N_{0}$ $\$ 10^{-4} \mathrm{GeV}$; i.e., if $M_{*} \sim 10^{5} \mathrm{GeV}$, then for $N_{0}$ $>10^{-4} \mathrm{GeV}$ there is no value of $m_{\phi}$ for which $N_{\mathrm{e} 2}<43$ and $\delta_{\mathrm{H}}=1.9 \times 10^{-5}$. The presence of such a small vacuum expectation value for the $N$ field and a negligible bare mass for the $\phi$ field is an extreme fine tuning in $4+d$ dimensions which is unlikely, so we will not pursue this possibility further.

Later we will examine whether it is possible to construct a satisfactory model where the cosmologically interesting density perturbations are produced during the first phase of inflation and have the correct amplitude, by employing an intermediate fundamental scale; however, for now we will continue to focus on the parameter values used in Refs. $[14,7]$.

For these parameters values the cosmologically interesting density perturbations are produced during the second phase of inflation, when both fields are dynamically important, and to calculate their amplitude we therefore need to employ the formula for multiple slow-rolling scalar fields [23]:

$$
\delta_{\mathrm{H}}^{2}=\frac{1}{75 \pi^{2}}\left(\frac{8 \pi}{M_{\mathrm{p}}^{2}}\right)^{3} V^{3}\left[\left(\frac{\mathrm{d} V}{\mathrm{~d} \phi}\right)^{2}+\left(\frac{\mathrm{d} V}{\mathrm{~d} N}\right)^{2}\right]^{-1} .
$$

The scales that we are interested in leave the Hubble radius very close to the end of the second period of inflation $\left[43 /\left(8.5 \times 10^{5}\right) \ll 1\right]$ when both fields are evolving rapidly and it is not possible to accurately follow their motion analytically. We therefore evolve the fields numerically and utilize Eq. (35) to evaluate $\delta_{\mathrm{H}}$. We can make several simple observations, however. After the beginning of the phase transition, but far from the end of inflation, $\mathrm{d} V / \mathrm{d} \phi \approx m_{\phi}^{2} \phi$ $\gg \mathrm{d} V / \mathrm{d} N$ so that $\delta_{\mathrm{H}}$ has the same value as prior to the phase transition, given by Eq. (33). The fields begin evolving more rapidly during the last 100 or so $e$-foldings of inflation with $\mathrm{d} V / \mathrm{d} N$ and $\mathrm{d} V / \mathrm{d} \phi$, which are of the same order of magnitude, increasing significantly, so that $\delta_{\mathrm{H}}$ decreases. We can make an order of magnitude estimate of $\delta_{\mathrm{H}}$ at the end of inflation, $\delta_{\mathrm{H}}(\epsilon=1)$ by pretending that only one of the fields is dynamically important and utilizing the single-field expression for $\delta_{\mathrm{H}}$ in terms of the first slow-roll parameter $\epsilon_{\phi}$ $\equiv\left(M_{P}^{2} / 16 \pi\right)\left(V^{\prime} / V\right)^{2}$ :

$$
\delta_{\mathrm{H}}=\frac{32}{75 M_{\mathrm{P}}^{4}} \frac{V}{\epsilon} .
$$

Inflation ends when $\epsilon=1$, so that ${ }^{1}$

$$
\delta_{\mathrm{H}}(\epsilon=1)<\frac{\lambda N_{0}^{2}}{2 M_{*} M_{\mathrm{P}}} .
$$

For the parameters used in Refs. [7,14] this gives $\delta_{\mathrm{H}}(\epsilon$ $=1)<4 \times 10^{-15}$. The large change in $\delta_{\mathrm{H}}$ towards the end of inflation is, along with the long duration of the second phase

\footnotetext{
${ }^{1}$ This is an upper limit since we are neglecting the change in $V$.
}

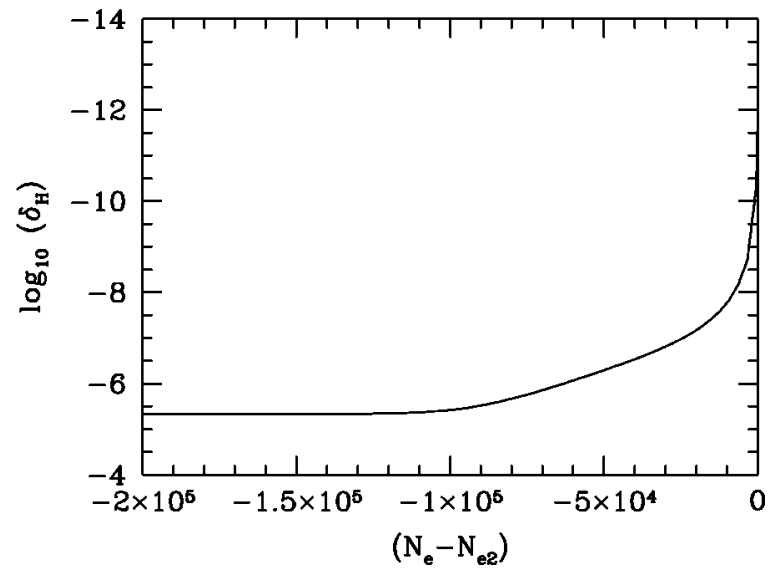

FIG. 2. The amplitude of the density perturbations, $\delta_{\mathrm{H}}$, as a function of minus the number of $e$-foldings before the end of inflation for $\lambda=1, N_{0}=M_{*} \sim 10^{5} \mathrm{GeV}$ and $m_{\phi}=10 \mathrm{GeV}$.

of inflation, a consequence of the extremely slow evolution of the fields at the beginning of the phase transition.

The variation of $\delta_{\mathrm{H}}$ with number of $e$-foldings before the end of inflation is shown in Fig. 2. We find that $43 e$-foldings before the end of inflation $\delta_{\mathrm{H}} \sim 3 \times 10^{-13}$, far smaller than required by the COBE normalization. We also find that $\delta_{\mathrm{H}}$ at the end of inflation is independent of $m_{\phi}$, as expected from Eq. (37). If $m_{\phi}$ is decreased, with the other parameters kept fixed, while $\delta_{\mathrm{H}}$ on scales which exit the Hubble radius well before the end of inflation is increased, as predicted by Eq. (35), the value $43 e$-foldings before the end remains stubbornly at $3 \times 10^{-15}$. Therefore for the parameters of the model proposed in Refs. $[7,11,14]$ it is not possible to produce density perturbations of the required size.

We will now examine whether it is possible to choose parameter values such that $N_{\mathrm{e} 2}<43$, so that the perturbations on the scales probed by COBE exit the Hubble radius during the first period of inflation, and $\delta_{\mathrm{H}}$, which is now given by Eq. (35), satisfies the COBE normalization. This can be achieved if the fundamental scale of gravity is at some intermediate scale. For instance, if $M_{*} \sim 10^{9} \mathrm{GeV}$, with $N_{0}$ $\sim 10^{4} \mathrm{GeV}$, and $m_{\phi} \sim 10^{-5} \mathrm{GeV}$, we find ${ }^{2}$ numerically that $N_{\mathrm{e} 2}<\mathcal{O}(10)$ and $\delta_{\mathrm{H}} \sim \mathcal{O}\left(10^{-5}\right)$. Note that even though $m_{\mathrm{N}}$ $>H$ for these parameter values, there is still a short second phase of inflation due to the small gradients, and consequent slow evolution, of the fields at the beginning of the phase transition. These parameters values should be considered as illustrative, as they are clearly not unique.

\section{IMPLICATIONS OF AN INTERMEDIATE FUNDAMENTAL SCALE}

We will now study some other implications of an intermediate fundamental scale for the model.

\footnotetext{
${ }^{2}$ The assumptions employed in the derivation of Eq. (32) break down for these parameter values.
} 


\section{A. Primordial black hole production}

For the set of parameter values considered in Refs. $[14,7,11] N_{0} \sim M_{*} \sim 10^{5} \mathrm{GeV}, m_{\phi} \sim 10 \mathrm{GeV}$, and $\lambda \sim g$ $\sim \mathcal{O}$ (1) the duration of the second phase of inflation is so long that the density perturbations on scales corresponding to the beginning of the phase transition, which may be large and lead to $\mathrm{PBH}$ overproduction $[16,17]$, remain well outside the Hubble radius today. For parameter values where there is a second phase of inflation with duration less than 43 $e$-foldings, these potentially large fluctuations will have reentered the Hubble radius by the present day and we need to worry about $\mathrm{PBH}$ overproduction. The production of $\mathrm{PBH}$ in extra dimensional scenarios at colliders and from high energy cosmic rays has been studied in great detail recently [24]. PBHs produced in the early universe, via the collapse of large density pertubarions, form via a completely different mechanism however. The evaporation of cosmological BHs in extra-dimensional scenarios has been examined [25], but the conditions for $\mathrm{PBH}$ formation in these scenarios are not known with any precision. We will therefore use the calculations of GLW to examine the order of magnitude constraint on the parameters of our model.

The amplitude of the fluctuations on scales corresponding to the beginning of the phase transition can be estimated as [17]

$$
\delta \equiv \frac{\delta \rho}{\rho} \approx \frac{4}{9 s},
$$

where

$$
s=-\frac{3}{2} \pm \sqrt{\frac{9}{4}+\frac{m_{\mathrm{N}}^{2}}{H^{2}}}
$$

If $\delta \ll 1$, then the present day density of PBHs will be negligible (see e.g. [26]). This is guaranteed if

$$
\frac{m_{\mathrm{N}}}{H}=\sqrt{\frac{3}{4 \pi}} \frac{M_{*}}{N_{0}} \gg 1 .
$$

This is precisely the condition usually given for inflation to end promptly at $\phi=\phi_{\mathrm{c}}[17]$, implying that a slow phase transition automatically leads to the formation of a nonnegligible population of PBHs. We have seen, however, that if the gradients of the fields are sufficiently small, a second phase of inflation, with non-negligible duration, can still occur even when this condition is marginally satisfied. We therefore conclude that to find whether PBHs are overproduced for the set of parameters suggested above would require a careful study of the formation of PBHs in extra dimensional scenarios and also an accurate calculation of the amplitude of the density perturbations produced at the beginning of the phase transition.

An attractive alternative would be to avoid the possibility of $\mathrm{PBH}$ overproduction entirely, by choosing parameter values for which the phase transition occurs rapidly and there is no second phase of inflation. In the extra dimensional model which we are studying, to ensure this, while producing den- sity perturbations of the required magnitude on cosmological scales, would require an even larger fundamental scale however.

\section{B. Baryogenesis}

For TeV scale quantum gravity with a normalcy temperature as low as $\sim 10 \mathrm{MeV}$, the only calculable and predictable model of baryogenesis has been given in Refs. [10,11]. Other avenues for baryogenesis exist in brane world scenarios where the standard model fields also propagate in the bulk. In this case it is possible to localize the fermionic wave function on a brane in such a way that a small overlap prevents fast proton decay [27]. Baryogenesis in these models has been explored in detail in Ref. [28]; however the model which we are studying is quite different, as it is assumed that the standard model fields are confined to the brane.

The general idea is based on the fact that there exists a gauge singlet which carries a $U(1)$ charge, which is dynamically broken at a scale governed by the Hubble expansion after the end of inflation [11]. This introduces a broken $C$ and $C P$ phase. As a result the real and imaginary parts of the $U(1)$ carrying field spiral and produce an initially asymmetric distribution of field quanta, which is transferred to the SM quarks and leptons via the dimensional-6 baryon number violating lepto-quark operator. Note that such an operator can also induce proton decay as long as the gauge singlet responsible for baryogenesis never develops any vacuum expectation value.

The additional second phase of inflation which we have found will not affect the dynamical mechanism of producing baryogenesis which was provided in Refs. [10,11]. However, in order to obtain the observed baryon asymmetry $\sim 10^{-10}$, the gauge singlet must have a large initial amplitude of order $\sim 10^{16} \mathrm{GeV}$. This could only be obtained if the singlet were promoted to the bulk along with the inflaton sector. Now, if we raise the fundamental scale to $\sim 10^{9} \mathrm{GeV}$, we certainly relax the stringent constraint on the normalcy temperature for two extra dimensions from $100 \mathrm{MeV}$ to $1 \mathrm{TeV}$ [10]. In this case our universe could afford to have a reheat temperature of the order of electroweak scale, which opens up the possibility of electroweak baryogenesis and leptogenesis. Note that the baryogenesis mechanism which was described above very briefly, and in detail in Refs. [10,11], would remain a viable option. Here again one has to promote the $U(1)$ carrying gauge singlet to the bulk along with the inflaton. This is because by raising the fundamental scale we are also raising the energy density stored in the inflaton sector, and in order to get the right baryon to photon ratio one then has to have a larger amplitude, comparable to the Planck scale now, for the gauge singlet.

\section{CONCLUSIONS}

We have studied in detail the dynamics of a hybrid inflationary model in the context of large extra dimensions, proposed in Ref. [14] and subsequently studied in Refs. $[7,10,11]$, where it is assumed that the inflaton sector is a gauge singlet residing in the higher dimensions. We have studied the entire gamut of the dynamical behavior of the 
coupled scalar fields. In particular we have shown that for a low fundamental scale, as studied in Refs. [7,11,14], there are in fact two distinct phases of inflation. The first phase of inflation has two parts, an initial period of radion dominated inflation, as the radion is stabilized via dynamical trapping in its own potential, followed by vacuum dominated inflation, as in standard hybrid models. At low energies, when the radion mass dominates the Hubble parameter, the radion begins oscillating around the minimum of its potential; however, if the bare mass of the radion is very small, $\lesssim 10^{-2} \mathrm{eV}$ in the case of two large extra dimensions and the fundamental scale $\mathcal{O}(\mathrm{TeV})$, then the radion density stored in the oscillations is not large enough to cause problems similar to the moduli problem.

For the parameter values considered natural in standard four dimensional hybrid inflation models, inflation ends rapidly, once the $\phi$ field reaches the critical value at which the false vacuum becomes unstable. For the parameters which are natural from an extra dimensional perspective [a fundamental scale $M_{*} \sim N_{0} \sim 10^{5} \mathrm{GeV}$ and fundamental coupling constants $\lambda, g \sim \mathcal{O}(1)]$ we find that the phase transition is slow, producing a second phase of inflation which lasts for around $10^{6} e$-foldings. Cosmologically interesting scales therefore exit the Hubble radius close to the end of this second period of inflation, and we find that the amplitude of the density perturbations on these scales is smaller than required by the COBE normalization unless the vacuum expectation value of the false vacuum field, $N_{0}$, is less than $10^{-4} \mathrm{GeV}$.

The only way around this obstacle is to try to shorten the second phase of inflation so that cosmologically interesting scales exit the Hubble radius before the phase transition. To do this we need a fundamental scale higher than $10^{5} \mathrm{GeV}$. We have found that, for instance, with the set of parameter values $M_{*} \sim 10^{9} \mathrm{GeV}, N_{0} \sim 10^{4} \mathrm{GeV}$ and $m_{\phi} \sim 10^{-5} \mathrm{GeV}$ density perturbations of the correct amplitude are produced. For this intermediate fundamental scale the constraint on the normalcy temperature is relaxed so that electroweak baryogenesis and leptogenesis become possible; furthermore, the baryogenesis mechanism provided in Ref. [10] is unaffected by the second phase of inflation and remains viable. If there is a second phase of inflation lasting less than $43 e$-foldings then the large density perturbations produced at the beginning of the phase transition will have re-entered the Hubble radius by the present day, and may lead to the overproduction of PBHs $[16,17]$. The formation criteria for PBHs in extra-dimensional scenarios have not yet been studied, so it is not possible to use the constraints on PBH abundance [26] to constrain the model parameters.

\section{ACKNOWLEDGMENTS}

A.M.G. was supported by PPARC and the Swedish Research Council. We thank Abdel Pérez Lorenzana for useful comments and Andrew Liddle for numerous useful discussions in the early stages of this work. A.M.G. acknowledges use of the Starlink computer system at Queen Mary, University of London.
[1] N. Arkani-Hamed, S. Dimopoulos, and G.R. Dvali, Phys. Lett. B 429, 263 (1998); Phys. Rev. D 59, 086004 (1999); I. Antoniadis, N. Arkani-Hamed, S. Dimopoulos, and G.R. Dvali, Phys. Lett. B 436, 257 (1998).

[2] For some early ideas see also I. Antoniadis, Phys. Lett. B 246, 377 (1990); I. Antoniadis, K. Benakli, and M. Quirós, ibid. 331, 313 (1994); K. Benakli, Phys. Rev. D 60, 104002 (1999); Phys. Lett. B 447, 51 (1999).

[3] C.P. Burgess, L.E. Ibanez, and F. Quevedo, Phys. Lett. B 447, 257 (1999).

[4] For experimental bounds see, for instance, T.G. Rizzo, Phys. Rev. D 59, 115010 (1999); G.F. Giudice, R. Rattazzi, and J.D. Wells, Nucl. Phys. B544, 3 (1999); E.A. Mirabelli, M. Perelstein, and M.E. Peskin, Phys. Rev. Lett. 82, 2236 (1999); J.L. Hewett, ibid. 82, 4765 (1999); V. Barger, T. Han, C. Kao, and R.J. Zhang, Phys. Lett. B 461, 34 (1999).

[5] C.D. Hoyle et al., Phys. Rev. Lett. 86, 1418 (2001).

[6] For a review see, for instance, A. Pérez-Lorenzana, in Particles and Fields: Ninth Mexican School, edited by Gerardo Herrera Carral and Lukas Nellen, AIP Conf. Proc. No. 562 (AIP, Melville, 2001), p. 53.

[7] A. Mazumdar and A. Pérez-Lorenzana, Phys. Lett. B 508, 340 (2001).

[8] K. Benakli and S. Davidson, Phys. Rev. D 60, 025004 (1999); L.J. Hall and D.R. Smith, ibid. 60, 085008 (1999); M. Fairbairn, Phys. Lett. B 508, 335 (2001); M. Fairbairn and L. M.
Griffiths, J. High Energy Phys. 02, 024 (2002).

[9] S. Hannestad and G.G. Raffelt, Phys. Rev. Lett. 88, 071301 (2002).

[10] R. Allahverdi, K. Enqvist, A. Mazumdar, and A. PérezLorenzana, Nucl. Phys. B618, 277 (2001).

[11] A. Mazumdar and A. Pérez-Lorenzana, Phys. Rev. D 65, 107301 (2002).

[12] D. Lyth, Phys. Lett. B 448, 191 (1999); G.R. Dvali and S.H.H. Tye, ibid. 450, 72 (1999); N. Kaloper and A. Linde, Phys. Rev. D 59, 101303 (1999); N. Arkani-Hamed et al., Nucl. Phys. B567, 189 (2000).

[13] A. Mazumdar, Phys. Lett. B 469, 55 (1999).

[14] R.N. Mohapatra, A. Pérez-Lorenzana, and C.A. de S. Pires, Phys. Rev. D 62, 105030 (2000).

[15] A.D. Linde, Phys. Lett. B 259, 38 (1991); Phys. Rev. D 49, 748 (1994).

[16] L. Randall, M. Soljačić, and A.H. Guth, Nucl. Phys. B472, 377 (1996).

[17] J. García-Bellido, A.D. Linde, and D. Wands, Phys. Rev. D 54, 6040 (1996).

[18] A.L. Berkin and K. Maeda, Phys. Rev. D 44, 1691 (1991).

[19] F.S. Accetta and P.J. Steinhardt, Phys. Rev. Lett. 67, 298 (1991).

[20] C.M. Will, Theory and Experimet in Gravitational Physics (Cambridge University Press, Cambridge, England, 1981).

[21] A.A. Starobinsky, in Current Topics in Field Theory, Quantum 
Gravity and Strings, Lecture Notes in Physics Vol. 206, edited by H.J. de Vega and N. Sanchez (Springer, Heidelberg, 1986), p. 107; A.S. Goncharov, A.D. Linde, and V.F. Mukhanov, Int. J. Mod. Phys. A 2, 561 (1987); A.D. Linde and A. Mezhlumian, Phys. Lett. B 307, 25 (1993); A.D. Linde, D.A. Linde, and A. Mezhlumian, Phys. Rev. D 49, 1783 (1994).

[22] E.F. Bunn, D. Scott, and M. White, Astrophys. J. Lett. 441, L9 (1995).

[23] M. Sasaki and E.D. Stewart, Prog. Theor. Phys. 95, 71 (1996).

[24] S.B. Giddings and S. Thomas, Phys. Rev. D 65, 056010 (2002); S. Dimopoulos and G. Landsberg, Phys. Rev. Lett. 87, 161602 (2001); J.L. Feng and A.D. Shapere, ibid. 88, 021303
(2002).

[25] P.C. Argyres, S. Dimopoulos, and J. March-Russell, Phys. Lett. B 441, 96 (1998); R. Emparan, G.T. Horowitz, and R.C. Myers, Phys. Rev. Lett. 85, 499 (2000).

[26] B.J. Carr, Astrophys. J. 201, 1 (1975); B.J. Carr, J.H. Gilbert, and J.E. Lidsey, Phys. Rev. D 50, 4853 (1994); A.M. Green and A.R. Liddle, ibid. 56, 6166 (1997).

[27] N. Arkani-Hamed, Y. Grossman, and M. Schmaltz, Phys. Rev. D 61, 115004 (2000).

[28] A. Masiero, M. Peloso, L. Sorbo, and R. Tabbash, Phys. Rev. D 62, 063515 (2000); T. Dent, hep-ph/0110318; D. Chung and T. Dent, hep-ph/0112360. 